\title{
The impact of rehabilitation on parents and caregivers of children with cerebral palsy: a systematic review
}

- A. Al-Mutayliq,

- G. Yeowell,

- F. Fatoye

http://dx.doi.org/10.1016/j.physio.2016.10.157

Relevance: Caregivers are central to achieving successful outcomes of rehabilitation for children with CP. Understanding its impact on caregivers may help to develop strategies to enable them to continue their caregiving role.

Purpose: The prevalence of cerebral palsy (CP) is 2.08 per 1000 live births. CP is associated with severe intellectual and physical impairments. Consequently, many children with $\mathrm{CP}$ require the assistance of a caregiver to accomplish daily life activities. Rehabilitation is a key intervention for children with $\mathrm{CP}$, and parents/caregivers of children with $\mathrm{CP}$ are central to achieving successful outcomes of rehabilitation. However, rehabilitation may place additional burdens on caregivers. To date, the impact of rehabilitation on parents and caregivers of children with CP is not well understood.

Objective: This systematic review examined the impact of rehabilitation on parents and caregivers of children with $\mathrm{CP}$.

Methods/analysis: Systematic searches of the following key databases were undertaken: AMED, CINAHL, MEDLINE, Science Direct, NHSEED, and PsycINFO. Inclusion criteria for the review were: randomised controlled trials or observational studies, articles published in English language in peer-reviewed journals, involved rehabilitation intervention for children with CP aged range 0 and 18 years, included parents and or caregivers. Articles were excluded if they were not published in English language, review, did not involve rehabilitation, and did not include parents or caregivers. To ensure that the relevant studies were selected, a random sample of $20 \%$ of the abstracts of all the identified studies were reviewed by two authors (AA, FF). A consensus method was used where there was disagreement, and an independent reviewer (GY) was consulted to make the decision regarding the final inclusion of the article in the review. Data were extracted using standard templates. The McMaster Critical Review Form for Quantitative Studies was used to assess the methodology of the studies.

Results: Following removal of duplicates, 11 studies out of 1153 met the inclusion criteria and were included in the review. The populations of the studies included 651 caregivers of children with CP. There were four randomised controlled trials and six observational studies using a range of rehabilitation interventions. The results indicated that rehabilitation had no significant effect on the caregivers' psychological stress or strain. General health was measured in only one study and it showed that rehabilitation had no significant effect on caregivers' health. However, one study found that there was a significant improvement in family empowerment following rehabilitation. In addition, parental and or caregivers' level of satisfaction with the process of rehabilitation for children with CP was high. 
Discussion and conclusions: Family empowerment improved significantly following rehabilitation for children with $\mathrm{CP}$. Given the complex nature of rehabilitation for children with $\mathrm{CP}$, the impact of rehabilitation on psychological wellbeing and physical health of parents/caregivers of children with this condition should be examined.

Impact and implications: There is limited evidence on the impact of rehabilitation on parents and caregivers of children with CP. Understanding this may help to put appropriate treatment strategies in place for parents and caregivers of children with this condition.

Funding acknowledgement: No funding was received in relation in relation to this study. 